Pacific Journal of Mathematic 


\section{WALLMAN RINGS}

\section{H. L. Bentley and B. J. TAylor}

In 1964 Frink defined a normal base. He hypothesized that every Hausdorff compactification of a Tychonoff space $X$ may be realized as a compactification $w(\mathscr{F})$ of Wallman type obtained from a normal base $\mathscr{F}$ on $X$, where $\mathscr{F}$ is the family of zero sets for some subring of $C(X)$. Later Biles formally defined a Wallman Ring on a Tychonoff space to be a subring of $C(X)$ whose zero sets form a normal base on $X$.

The problem in this paper is to study examples of Wallman Rings and develop properties of Wallman Rings. For a locally compact space with a given compactification and a certain type of retract map, a Wallman Ring is defined which induces the given compactification.

General algebraic and topological properties of Wallman Rings are considered. Among the results obtained are "Every Wallman Ring is equivalent to one which contains all rational constant functions" and "An ideal of a Wallman Ring which is itself a Wallman Ring is equivalent to the superring."

I. Introduction. In $1938 \mathrm{H}$. Wallman [23] gave a method for associating a compact $T_{1}$-space $w(\mathscr{F})$ with a distributive lattice $\mathscr{F}$; $w(\mathscr{F})$ is the space of all $\mathscr{F}$-ultrafilters and the topology of $w(\mathscr{F})$ has a base for closed sets a lattice $\mathscr{F}^{*}$ which is isomorphic to the lattice $\mathscr{F}$. Wallman applied this procedure to the case when $\mathscr{F}$ is the lattice of all closed subsets of a $T_{1}$-space $X$ to arrive at a $T_{1}$ compactification $w(\mathscr{F})$ of $X$ which is now called "the Wallman Compactification" of $X$.

Several later mathematicians applied Wallman's construction to certain types of lattices which are sublattices of the lattice of all closed sets of a $T_{1}$-space. Among these were Sanin [18], Banaschewski [3], and Frink [12]. These techniques give rise to certain classes of compactifications. In 1964, Frink [12] asked whether each Hausdorff compactification of a Tychonoff space $X$ can be realized as a compactification $w(\mathscr{F})$ of Wallman type obtained from a normal base $\mathscr{F}$ for the closed sets of $X$. (A normal base is a lattice which is a base for closed sets and which satisfies certain separation properties.) This question remains unanswered to the present day, but a great deal of effort has been exerted by many mathematicians in an endeavor to solve the problem.

Partial solutions have been obtained, e.g. Steiner and Steiner have shown that any product of compact metric spaces is a Wallman type 
compactification (determined by some normal base) of any of its dense subspaces. It has become customary to call a compactification of the form $w(\mathscr{F})$, where $\mathscr{F}$ is some normal base, a "Wallman compactification" of $X$ and we shall use this terminology in the sequel.

Frink observed that the family $Z(X)$ of all zero sets of continuous real valued functions on a Tychonoff space $X$ is a normal base on $X$ which gives rise to a compactification $w(Z(X))$ equivalent to the Stone-Cech compactification $\beta X$ of $X$. He also observed that if $Y$ is a given compactification of $X$, then $Z(E(X, Y))$, the zero sets of continuous real valued functions on $X$ which are continuously extendible to $Y$, is a normal base. Biles [8] later called a subring $\mathscr{A}$ of the ring $C(X)$, of all real valued continuous functions on $X$, a Wallman ring on $X$ whenever $Z(\mathscr{A})$, the zero sets of functions in $\mathscr{A}$, form a normal base.

Frink wondered whether every Hausdorff compactification of a Tychonoff space $X$ is of the form $w(Z(\mathscr{A}))$ where $\mathscr{A}$ is some Wallman ring on $X$. This question is still unanswered although many partial results have been obtained.

Biles [8] studied relationships between the Gelfand and the Wallman compactifications determined by a Wallman ring $\mathscr{A}$ on a Tychonoff space $X$.

In this paper we study examples of Wallman rings and develop properties of Wallman rings.

In §IV we will consider a locally compact space and a compactification of that space such that there is a certain type of retract map on the compactification. We establish a Wallman ring on this locally compact space which induces the given compactification. From this result we are able to define a Wallman ring which yields the Alexandroff compactification and a Wallman ring on the open unit disc which induces a compactification equivalent to the closed unit disc.

In section III we consider general properties of Wallman rings on spaces with more than one element. We find that a Wallman ring cannot be an integral domain; that every Wallman ring is equivalent to one which is inverse closed; that an ideal of a Wallman ring which is itself a Wallman ring is equivalent to the larger ring. We also examine the relationship between a Wallman ring being the direct sum of nontrivial ideals and its associated compactification being disconnected. We present some results linking Wallman rings to sublattices of $C(X)$, and we pose the question: "Is every Wallman ring on $X$ equivalent to one which is a sublattice of $C(X)$ ?"

II. Preliminary notations and definitions. Throughout this paper, all topological spaces are Tychonoff (completely regular and Hausdorff), and contain at least two points. If $X$ is a topological space, 
then a compact space $Y$ is said to be a compactification of $X$ if there is a homeomorphism $h$ from $X$ into $Y$ such that $h[X]$ is dense in $Y$. The function $h$ is called an embedding. To simplify notation, embeddings will be taken to be inclusions.

We will use the customary ordering of compactifications: $Y_{1} \leqq Y_{2}$ if there is a continuous map $f: Y_{2} \rightarrow Y_{1}$ which leaves $X$ pointwise fixed (i.e. $f(x)=x$ for all $x \in X)$. Also, $Y_{1}$ and $Y_{2}$ are equivalent $\left(Y_{1} \cong Y_{2}\right)$ if $Y_{1} \leqq Y_{2}$ and $Y_{2} \leqq Y_{1}$. As is well known, $Y_{1} \cong Y_{2}$ if and only if there is a homeomorphism $f: Y_{1} \rightarrow Y_{2}$ which leaves $X$ pointwise fixed.

We will use the following notation throughout the paper.

$\mathcal{N}$ - the set of natural numbers

$\mathscr{R}$ - the field of real numbers

$C(X)$ - the ring of all real valued continuous functions on the space $X$.

$C^{*}(X)$ - the ring of all bounded functions in $C(X)$.

$\mathscr{A}^{*}$ - the subset of bounded functions of a collection $\mathscr{A} \subseteq C(X)$, $\mathscr{A}^{*}=\left\{f \in \mathscr{A}: f \in C^{*}(X)\right\}$

$Z(f)$ - the zero set of a real valued function $f$ on $X, Z(f)=$ $\{x \in X: f(x)=0\}$.

$Z[\mathscr{A}]$ - the zero sets of a collection $\mathscr{A}$ of real valued functions on $X, Z[\mathscr{A}]=\{Z(f): f \in \mathscr{A}\}$.

$Z(X)$ - the zero sets of $C(X), Z(X)=Z[C(X)]=Z\left[C^{*}(X)\right]$.

For basic concepts regarding the ring $C(X)$, we refer the reader to Gillman and Jerison [13].

Following the terminology of Biles [8] and Frink [12] we give the following definitions.

2.1. Definition. If $\mathscr{F}$ is a collection of subsets of $X$, then $\mathscr{F}$ is a lattice on $X$ if:

(1) $\phi, X \in \mathscr{F}$

(2) If $A, B \in \mathscr{F}$, then $A \cap B \in \mathscr{F}$ and $A \cup B \in \mathscr{F}$.

2.2. Definition. The lattice $\mathscr{F}$ on $X$ is a normal base on $X$ if $\mathscr{F}$ is:

(1) a base for the closed subsets of $X$,

(2) a disjunctive lattice on $X$ (i.e. if $A \in \mathscr{F}$ and $x \in X-A$, then there exists $B \in \mathscr{F}$ such that $x \in B$ and $A \cap B=\phi$ ),

(3) a normal lattice on $X$ i.e. for each $A, B \in \mathscr{F}$ such that $A \cap B=\phi$, there exists $C, D \in \mathscr{F}$ such that $A \cap D=\phi, B \cap C=\phi$ and $C \cup D=X$.

The space $w(\mathscr{F})$ consisting of the set of all $\mathscr{F}$-ultrafilters on $X$ is a Hausdorff compactification of $X$. The topology of $w(\mathscr{F})$ is defined as 
follows. If $A \in \mathscr{F}, A^{*}$ is the set of all $\mathscr{F}$-ultrafilters having $A$ as a member. A base for the closed subsets of $w(\mathscr{F})$ is the set of all $A^{*}$ such that $A \in \mathscr{F}$.

$X$ is embedded in $w(\mathscr{F})$ by the map which sends a point $x \in X$ into the $\mathscr{F}$-ultrafilter consisting of all $\mathscr{F}$-sets which contain $x$.

2.3. Definition. $\quad w(\mathscr{F})$ is said to be a Wallman compactification of $X$.

As was mentioned earlier a subring $\mathscr{A}$ of $C(X)$ is a Wallman ring on $X$ if $Z[\mathscr{A}]$ is a normal base on $X$. So if $\mathscr{A}$ is a Wallman ring on $X$, then $w(Z[\mathscr{A}])$ is a Wallman compactification of $X$.

We will content ourselves here with the above statements on Wallman compactifications. The reader is referred to the literature (e.g. Frink [12], Biles [8], Steiner [19], Alo and Shapiro [2]) for proofs of the above statements.

Being interested in ordering of compactifications, we are led to the following concept which is due to Steiner [19].

2.4. Definition. Let $\mathscr{F}$ and $\mathscr{G}$ be families of sets. Then:

(1) $\mathscr{F} \leqq \mathscr{G}$ ( $\mathscr{G}$ separates $\mathscr{F})$ if and only if for each $F_{1}, F_{2} \in \mathscr{F}$, $F_{1} \cap F_{2}=\phi$ implies there exist $G_{1}, G_{2} \in \mathscr{G}$ such that $F_{1} \subseteq G_{1}, F_{2} \subseteq G_{2}$ and $G_{1} \cap G_{2}=\phi$.

(2) $\mathscr{F} \cong \mathscr{G}(\mathscr{F}$ is equivalent to $\mathscr{G})$ if and only if $\mathscr{F} \leqq \mathscr{G}$ and $\mathscr{G} \leqq \mathscr{F}$.

2.5. THEOREM. The relation "ミ" defined in 2.4 is transitive and reflexive. The relation " $\cong$ " is an equivalence relation.

We will now look at an application of this concept. By a sublattice of $C(X)$ we mean a subset of $C(X)$ which contains the supremum and infimum of each pair of its elements. By a closed subring of $C(X)$ we mean a subring of $C(X)$ which is closed in the uniform topology on $C(X)$.

2.6. Definition. $\mathscr{A}$ is an inverse closed subset of $C(X)$ if and only if for each $f \in \mathscr{A}$ and for each $g \in \mathscr{A}$ such that $Z(g)=\phi, f / g \in \mathscr{A}$.

2.7. Theorem. Let $\mathscr{A}$ be a Wallman subring and sublattice of $C(X)$, then $f, g \in \mathscr{A}$ implies $\{x \in X: f(x) \leqq g(x)\} \in Z[\mathscr{A}]$.

Proof. $\{x \in X: f(x) \leqq g(x)\}=Z((f-g) \vee 0)$.

In a restricted situation, the following theorem gives some insight into the separating relation of Steiner defined in 2.4. This theorem also is closely related to generalizations of the Stone-Weierstrass Theorem (for details see Taylor and Bentley [22]). 
2.8. THEOREM. Let $\mathscr{A}$ be a sublattice of $C(X)$ which contains the constant functions. Let $\mathscr{B}$ be an inverse closed subset of $C(X)$ which is also a closed subring of $C(X)$. Then $Z[\mathscr{A}] \leqq Z[\mathscr{B}]$ if and only if $\mathscr{A}^{*} \subseteq \mathscr{B}$.

Proof. Half of the proof is obvious. We will show only the other half. We borrow our method of proof from Hager [14].

Let $g \in \mathscr{A}^{*}$. There is a positive real number $r$ such thät $|g| \leqq r$. Choose $\epsilon>0$. Then there is a natural number $n$ such that $1 / n<\epsilon / 2 r$. Let $I=\{-n-1,-n, \cdots,-1,0,1, \cdots, n-1\}$.

For $i \in I$, let

$$
F_{i}=\{x \in X: g(x) \leqq i r / n\} \cup\{x \in X:(i+2) r / n \leqq g(x)\} .
$$

Then $F_{i} \in Z[\mathscr{A}]$ and $\cap_{i \in I} F_{i}=\phi$. As was shown by H. L. Bentley [4] there are functions $f_{i} \in \mathscr{B}$ for each $i \in I$ such that $F_{i} \subseteq Z\left(f_{i}\right)$ and $\cap_{i \in I} Z\left(f_{i}\right)=\phi$. For each $j \in I$, let

$$
h_{j}=\frac{f_{j}^{2}}{\sum_{i \in I} f_{i}^{2}} .
$$

So $\Sigma_{j \in I} h_{j}=1$ and since $\mathscr{B}$ is inverse closed $h_{j} \in \mathscr{B}$ for each $j \in I$.

Let $u=\sum_{i \in I} i r h_{i} / n$. Since $\mathscr{B}$ is inverse closed, is a closed subring of $C(X)$, and contains a function whose zero set is empty, namely $\Sigma_{i \in I} f_{i}^{2}$, $\mathscr{B}$ contains all the constant functions. Therefore $u \in \mathscr{B}$.

Now let $x \in X$ and let $j$ be as small as possible so that $x \notin F_{j} ; x$ cannot be in each of the $F_{i}$ since $\cap_{i \in I} F_{i}=\phi . \quad x \notin F_{j}$ implies $j r / n<$ $g(x)<(j+2) r / n$ so $|g(x)-(j+1) r / n|<r / n$ and

$$
\begin{aligned}
& x \in\left(\cap\left\{F_{i}: i \in I, i<j\right\}\right) \cap\left(\cap\left\{F_{i}: i \in I, i>j+1\right\}\right) . \\
& \text { Now } \quad u(x)=\sum_{i \in I} \frac{i}{n} r h_{i}(x) \\
& =\frac{j}{n} r h_{j}(x)+\frac{j+1}{n} r h_{j+1}(x) \\
& =\frac{j}{n} r\left(h_{j}(x)+h_{j+1}(x)\right)+\frac{r}{n} h_{j+1}(x) \\
& \left.=\frac{j}{n} r\left(\sum_{i \in I} h_{i}(x)\right)+\frac{r}{n} h_{j+1}(x)\right) \\
& =\frac{j}{n} r+\frac{r}{n} h_{j+1}(x) \text {. }
\end{aligned}
$$


Since $0 \leqq h_{j+1}(x) \leqq 1$ this yields

$$
\frac{j}{n} r \leqq u(x) \leqq \frac{j}{n} r+\frac{r}{n}=\frac{j+1}{n} r .
$$

Therefore $|u(x)-(j+1) r / n| \leqq r / n$ and

$$
|g(x)-u(x)| \leqq\left|g(x)-\frac{j+1}{n}\right|+\left|u(x)-\frac{j+1}{n}\right| \leqq \frac{2 r}{n}<\epsilon .
$$

For $\epsilon>0$, we have shown there is a function $u \in \mathscr{B}$ such that $|g(x)-u(x)|<\epsilon$ for each $x \in X$. Therefore, since $\mathscr{B}$ is closed in $C(X)$, $g \in \mathscr{B}$.

The following theorem is due to A. K. Steiner and E. F. Steiner [21].

2.9. THEOREM. If $\mathscr{F}$ and $\mathscr{G}$ i re normal bases on $X$, then:

(1) $\mathscr{F} \leqq \mathscr{G}$ if and only if $w(\mathscr{F}) \leqq w(\mathscr{G})$.

(2) $\mathscr{F} \cong \mathscr{G}$ if and only if $w(\mathscr{F}) \cong w(\mathscr{G})$.

2.10. THEOREM. If $\mathscr{F} \cong \mathscr{G}$ ind $\mathscr{F}$ is normal, then $\mathscr{G}$ is normal.

Proof. Let $G_{1}$ and $G_{2}$ be disjoint elements of $\mathscr{G} . \quad \mathscr{G} \leqq \mathscr{F}$ implies there are sets $F_{1}, F_{2} \in \mathscr{F}$ such that $G_{1} \subseteq F_{1}, G_{2} \subseteq F_{2}$ and $F_{1} \cap F_{2}=\phi$. Since $\mathscr{F}$ is normal there exist $F_{1}^{\prime}, F_{2}^{\prime} \in \mathscr{F}$ such that $F_{1} \cap F_{2}^{\prime}=\phi$, $F_{2} \cap F_{1}^{\prime}=\phi$ and $F_{1}^{\prime} \cup F_{2}^{\prime}=X$. Now, since $\mathscr{G}$ separates $\mathscr{F}$ there exist $G_{1}^{\prime}$, $G_{2}^{\prime}, G_{a}, G_{b} \in$ (S) such that $F_{1} \subseteq G_{a}, F_{2}^{\prime} \subseteq G_{2}^{\prime}, F_{2} \subseteq G_{b}, F_{1}^{\prime} \subseteq G_{1}^{\prime}, G_{a} \cap G_{2}^{\prime}=$ $\phi$ and $G_{b} \cap G_{1}^{\prime}=\phi$.

Now $G_{1} \subseteq F_{1} \subseteq G_{a}$ and $G_{a} \cap G_{2}^{\prime}=\phi$ implies $G_{1} \cap G_{2}^{\prime}=\phi$. $G_{2} \subseteq F_{2} \subseteq G_{b}$ and $G_{b} \cap G_{1}^{\prime}=\phi$ implies $G_{2} \cap G_{1}^{\prime}=\phi$. and $F_{1}^{\prime} \subseteq G_{1}^{\prime}$ and $F_{2}^{\prime} \subseteq G_{2}^{\prime}$ implies $G_{1}^{\prime} \cup G_{2}^{\prime}=X$. Therefore $\mathscr{G}$ is normal.

2.11. THEOREM. If $\mathscr{F}$ is a disjunctive base for the closed sets of $X$, $\mathscr{G}$ is a collection of closed sets of $X$ and $\mathscr{F} \leqq \mathscr{G}$, then $\mathscr{G}$ is a disjunctive base for the closed sets of $X$.

Proof. $X$ and $\phi \in \mathscr{G}$ since $X$ and $\phi$, as elements of $\mathscr{F}$, must be separated by elements of $\mathscr{G}$.

If $C$ is a closed subset of $X$ and $x$ is an element of $X$ not in $C$, then there exists $F_{1} \in \mathscr{F}$ such that $C \subseteq F_{1}$ and $x \notin F_{1}$. Consequently there exists $F_{2} \in \mathscr{F}$ such that $x \in F_{2}$ and $F_{1} \cap F_{2}=\phi$. 
Since $\mathscr{F} \leqq \mathscr{G}$, there are $G_{1}, G_{2} \in \mathscr{G}$ such that $F_{1} \subseteq G_{1}, F_{2} \subseteq G_{2}$ and $G_{1} \cap G_{2}=\phi$. This implies $\mathscr{G}$ is a base for the closed sets of $X$ since $x \notin G_{1}$ and $C \subseteq G_{1}$.

If $G \in \mathscr{G}$ and $x \notin G$, then as above there are $F_{1}, F_{2} \in \mathscr{F}$ such that $x \in F_{2}, G \subseteq F_{1}$ and $F_{1} \cap F_{2}=\phi$; and consequently disjoint $G_{1}, G_{2} \in \mathscr{G}$ such that $x \in G_{2}, G \subseteq G_{1}$. Since $x \in G_{2}$ and $G \cap G_{2}=\phi$, $\mathscr{G}$ is a disjunctive base for the closed sets in $X$.

2.12. TheOREM. If $\mathscr{F}$ is a normal base on $X, \mathscr{G}$ is a lattice of closed subsets of $X$ and $\mathscr{F} \cong \mathscr{G}$, then $\mathscr{G}$ is a normal base on $X$ and $w(\mathscr{F}) \cong w(\mathscr{G})$.

We will now translate this result from normal bases to Wallman rings.

2.13. Definition. If $\mathscr{A}$ and $\mathscr{B}$ are subrings of $C(X)$ then:

(1) $\mathscr{A} \leqq \mathscr{B}$ if and only if $Z[\mathscr{A}] \leqq Z[\mathscr{B}]$.

(2) $\mathscr{A} \cong \mathscr{B}$ if and only if $\mathscr{A} \leqq \mathscr{B}$ and $\mathscr{B} \leqq \mathscr{A}$.

2.14. THEOREM. If $\mathscr{A} \_$nd $\mathscr{B}$ are Wallman subrings of $C(X)$ then :

(1) $\mathscr{A} \leqq \mathscr{B}$ if and only if $w(Z[\mathscr{A}]) \leqq w(Z[\mathscr{B}])$

(2) $\mathscr{A} \cong \mathscr{B}$ if and only if $w(Z[\mathscr{A}]) \cong w(Z[\mathscr{B}])$.

2.15. THEOREM. $\mathscr{A}$ is a Wallman ring on $X, \mathscr{B}$ is a subring of $C(X)$ and $\mathscr{A} \cong \mathscr{B}$, then $\mathscr{B}$ is a Wallman ring on $X$ and $w(Z[\mathscr{A}]) \cong$ $w(Z[\mathscr{B}])$.

\section{Properties of Wallman Rings and Some} Questions. Since a Wallman ring is a ring in the usual algebraic sense, it is natural for us to investigate which properties of rings Wallman rings possess. Along this line we have discovered that a Wallman ring cannot be an integral domain, and that each Wallman ring is equivalent to a Wallman ring which is inverse closed. We have investigated the problem of when a Wallman ring is equivalent to a Wallman ring which is a sublattice of $C(X)$, but have only partial results.

We also investigated relationships between algebraic properties of Wallman rings and topological properties of the induced compactifications. Our main result along this line is one involving the relationship between a Wallman ring being the direct sum of nontrivial ideals and the induced compactification being disconnected.

Our first result is the following. 
3.1. Theorem. A Wallman ring cannot be an integral domain.

Proof. Let $\mathscr{A}$ be a Wallman ring on a space $X$ and let $x$ and $y$ be distinct elements of $X$. Since $Z[\mathscr{A}]$ is a disjunctive base for the closed sets of $X$, there are functions $f$ and $g \in \mathscr{A}$ such that $x \in Z(f), y \in Z(g)$ and $Z(f) \cap Z(g)=\phi$. By the normality of $Z[\mathscr{A}]$ there are functions $f^{\prime}$ and $g^{\prime} \in \mathscr{A}$ such that $Z(f) \cap Z\left(g^{\prime}\right)=\phi, \quad Z(g) \cap Z\left(f^{\prime}\right)=\phi \quad$ and $Z\left(f^{\prime}\right) \cup Z\left(g^{\prime}\right)=X$. Now we have $f^{\prime}(y) \neq 0, g^{\prime}(x) \neq 0$ but $f^{\prime} g^{\prime}$ is the zero function.

We will now show that every Wallman ring is equivalent to a Wallman ring which is inverse closed and therefore to a Wallman ring which contains all the rational constants.

3.2. Lemma. Every Wallman ring contains at least two functions whose zero sets are pairwise disjoint and nonempty, and a function whose zero set is empty.

Proof. Let $\mathscr{A}$ be a Wallman ring over $X$. Let $x$ and $y$ be distinct elements of $X$. Then there are functions $f_{1}$ and $f_{2} \in \mathscr{A}$ such that $x \in Z\left(f_{1}\right), y \in Z\left(f_{2}\right)$ and $Z\left(f_{1}\right) \cap Z\left(f_{2}\right)=\phi$. Then $f_{1}^{2}+f_{2}^{2}$ is a function from $\mathscr{A}$ whose zero set is empty.

3.3. THEOREM. Every Wallman ring is equivalent to a Wallman ring which is inverse closed.

Proof. Let $\mathscr{A}$ be a Wallman ring on $X$ and let $\mathscr{B}=\{f / g: f, g \in \mathscr{A}$, $Z(g)=\phi\}$. If $f \in \mathscr{A}$ then $Z(f)=Z(f / g)$ where $g$ is some function from $\mathscr{A}$ such that $Z(g)=\phi$; so $Z[\mathscr{A}] \subseteq Z[\mathscr{B}]$. Similarly $Z[\mathscr{B}] \subseteq Z[\mathscr{A}]$. $\mathscr{B}$ is a subring of $C(X)$ and $\mathscr{A} \cong \mathscr{B}$ so by Theorem $2.15 \mathscr{B}$ is a Wallman ring and $w(Z[\mathscr{A}]) \cong w(Z[\mathscr{B}])$.

3.4. COROLlary. Every Wallman ring is equivalent to a Wallman ring which contains all the rational constants.

Proof. Let $\mathscr{A}$ be a Wallman ring on $X$. Let $\mathscr{B}$ be the Wallman ring $\{f / g: f, g \in \mathscr{A}, Z(g)=\phi\}$. Let $g \in \mathscr{A}$ such that $Z(g)=\phi$. Then if $m$ and $n$ are integers, $n \neq 0, m g / n g \in \mathscr{B}$ and $(m g / n g)=(m / n)$.

From this proof we observe that every inverse closed Wallman ring contains all the rational constant functions.

The Wallman ring $C^{*}(X)$ is equivalent to the inverse closed Wallman ring $C(X)$, for any given space $X . C^{*}(X)$ itself need not be inverse closed. 
3.5. Definition. If $Y$ is a compactification of $X$, then $E(X, Y)$ is the set of all real valued continuous functions on $X$ which are continuously extendable to $Y$.

Frink [12] was the first to observe that $E(X, Y)$ is a Wallman ring on $X$. Proofs of this were later given by Hager [14] and Biles [8].

In the next theorem we give conditions for $C^{*}(X)$ to be inverse closed.

3.6. THEOREM. The following are equivalent:

(1) $X$ is pseudocompact,

(2) Every nonempty zero set of $\beta X$ meets $X$,

(3) For any compactification $Y$ of $X$, every nonempty zero set of $Y$ meets $X$,

(4) For any compactification $Y$ of $X, E(X, Y)$ is inverse closed,

(5) $C^{*}(X)$ is inverse closed.

Proof. $1 \Rightarrow 2$. Let $f \in C(\beta X)$ and suppose $Z(f \mid X)=\phi$. Then $(1 / f \mid X) \in C(X)=C^{*}(X)$. So So there exists $g \in C(\beta X)$ such that $g \mid X=(1 / f \mid X)$. Now $(g f) \mid X=1$ so $g f=1$ and $Z(f)=\phi$. So $Z(f \mid X)=$ $\phi$ implies $Z(f)=\phi$ or equivalently $Z(f) \neq \phi$ implies $Z(f \mid X) \neq \phi$.

$2 \Rightarrow 3$. Let $\alpha$ be a continuous mapping of $\beta X$ into $Y$ which leaves $X$ pointwise fixed. The existence of such a function is guaranteed by Stone's Theorem [14]. Let $f \in C(Y)$ such that $Z(f) \neq \phi$ and suppose $Z(f \mid X)=\phi$. Let $g=f \mid X . \quad g \in C(X)$ so $g$ has an extension to a continuous function $g^{\beta}$ in $C(\beta X), g^{\beta}=f \circ \alpha$. Since $Z(g)=\phi, Z\left(g^{\beta}\right)=$ $\phi$. Let $x \in Z(f)$. There exists $y \in \beta X$ such that $x=\alpha(y)$. Therefore $0=f(x)=f(\alpha(y))=g^{\beta}(y)$, which contradicts the fact that $Z\left(g^{\beta}\right)=\phi$. Therefore $Z(f) \neq \phi$ implies $Z(f \mid X) \neq \phi$.

$3 \Rightarrow 4$. . Let $f \in E(X, Y)$ such that $Z(f)=\phi$. There is a function $g \in C(Y)$ such that $f=g \mid X$. Since $Z(g \mid X)=\phi, Z(g)=\phi$ and $1 / g \in C(Y)$. Now $1 / f=1 / g \mid X$, so $1 / f \in E(X, Y)$ and $E(X, Y)$ is inverse closed.

$$
4 \Rightarrow 5 . \quad C^{*}(X)=E(X, \beta X)
$$

$5 \Rightarrow 1$. Let $f \in C(X)$. Let $g=|f| \vee 1$. $f$ will be bounded if and only if $g$ is. $Z(g)=\phi$ so $1 / g \in C(X)$. But $|1 / g|=1 / g \leqq 1$, so $1 / g \in C^{*}(X)$. Of course $Z(1 / g)=\phi$. Therefore $g=1 /(1 / g) \in C^{*}(X)$, and $C(X)=C^{*}(X)$.

Parts of this theorem are problems in Gillman and Jerison. $1 \Leftrightarrow 2$ is problem $6 \mathrm{I}, 1 \Leftrightarrow 5$ is problem $15 \mathrm{Q}$.

We now investigate what happens when $\mathscr{A}$ is a Wallman ring and $\mathscr{B}$ is an ideal of $\mathscr{A}$. 
3.7. Theorem. If $\mathscr{A}$ is a Wallman ring on $X, \mathscr{B}$ is an ideal of $\mathscr{A}$ and $Z[\mathscr{B}]$ is a disjunctive base on $X$, then $\mathscr{A} \leqq \mathscr{B}$.

Proof. Let $f$ and $g \in \mathscr{A}$ such that $Z(f) \cap Z(g)=\phi$. Assume $Z(f) \neq \phi$ and $Z(g) \neq \phi$ since otherwise the conclusion follows from $X \in Z[\mathscr{B}]$ and $\phi \in Z[\mathscr{B}]$. Let $x \in Z(f)$. Then $x \notin Z(g)$ so there is a function $f_{x} \in \mathscr{B}$ such that $x \in Z\left(f_{x}\right)$ and $Z\left(f_{x}\right) \cap Z(g)=\phi$. Now let $y \in Z(g) ; y \notin Z\left(f f_{x}\right)$ so there is a function $g_{y} \in \mathscr{B}$ such that $y \in Z\left(g_{y}\right)$ and $Z\left(g_{y}\right) \cap\left(f f_{x}\right)=\phi$. Consequently $f f_{x}$ and $g g_{y}$ are functions from $\mathscr{B}$ whose zero sets separate the zero sets of $f$ and $g$.

3.8. COROllaRY. If $\mathscr{A}$ and $\mathscr{B}$ are Wallman rings on $X$ and $\mathscr{B}$ is an ideal of $\mathscr{A}$ then $\mathscr{A} \cong \mathscr{B}$, i.e., $w(Z[\mathscr{A}]) \cong e(Z[\mathscr{B}])$.

The next corollary tells us that a Wallman ring cannot be an ideal of $C(X)$ unless the Wallman ring is $C(X)$ itself.

3.9. Corollary. If $\mathscr{A}$ is an inverse closed Wallman ring on $X$, then $\mathscr{A}$ has no proper nontrivial ideals whose zero sets are disjunctive; consequently $\mathscr{A}$ has no proper ideals which are Wallman rings.

Proof. Let $\mathscr{B}$ be an ideal of $\mathscr{A}$ such that $Z[\mathscr{B}]$ is disjunctive. By Lemma 3.2 there are at least two disjoint non-empty zero sets of $\mathscr{A}$, say $F_{1}$ and $F_{2}$. By Theorem 3.7 there are functions $f_{1}$ and $f_{2} \in \mathscr{B}$ such that $F_{1} \subseteq Z\left(f_{1}\right), F_{2} \subseteq Z\left(f_{2}\right)$ and $Z\left(f_{1}\right) \cap Z\left(f_{2}\right)=\phi$. Let $f=f_{1}^{2}+f_{2}^{2} \cdot f \in \mathscr{R}$ and $Z(f)=\phi$. Since $\mathscr{A}$ is inverse closed $g / f \in \mathscr{A}$ for each $g \in \mathscr{A}$. Therefore $g=f g / f \in \mathscr{B}$ for each $g \in \mathscr{A}$. The only nontrivial ideal of $\mathscr{A}$ whose zero sets are disjunctive is $\mathscr{A}$ itself.

If we were to eliminate the hypothesis in 3.9 that $\mathscr{A}$ be inverse closed, then the conclusion of the corollary would not necessarily follow, as is illustrated in this example.

3.10. Example. Let $X=(0,1], \mathscr{A}=C^{*}(0,1]$,

$$
\mathscr{B}=\left\{f \in \mathscr{A}: \lim _{x \rightarrow 0} f(x)=0\right\}
$$

Then $\mathscr{A}$ is a Wallman ring on $X$ which is not inverse closed, $\mathscr{B}$ is a proper ideal of $\mathscr{A}$ and as we shall now show, $Z(\mathscr{B})$ is disjunctive. Let $f \in \mathscr{B}$ and let $y \in X-Z(f)$. Then for some $a \in X, 0<a<y$ and $[a, y] \cap Z(f)=\phi$. Let $H=([a, 1] \cap Z(f)) \cup\{a\}$. Then $y \notin H$ and $H$ is closed in $[a, 1]$. So, there exists a function $g \in C([a, 1])$ so that $g(y)=0$ and $g(H)=1$. Observe that $g(a)=1$. Extended $g$ to a function $h \in \mathscr{B}$ 
by defining $h(x)=x / a$ for each $x \in(0, a)$. Clearly $y \in Z(h)$ and $Z(f) \cap Z(h)=\phi$ so that $Z(\mathscr{B})$ is disjunctive.

We now divert our attention to the topology of a space with relation to the zero sets of a Wallman ring on the space and observe that the following properties hold.

3.11. THEOREM. If $\mathscr{A}$ is a Wallman ring on $X$, then every neighborhood of a point $x \in X$ contains a $Z[\mathscr{A}]$ - neighborhood of $x$.

Proof. Let $A$ be a neighborhood of $x, x \in X .\{X-Z(f): f \in \mathscr{A}\}$ is a base for the open sets of $X$. So for some $f \in \mathscr{A}, x \in(X-Z(f)) \subseteq A$. Since $Z[\mathscr{A}]$ is disjunctive, there is a function $g \in \mathscr{A}$ such that $g(x)=0$ and $Z(g) \cap Z(f)=\phi$. By the normality of $Z[\mathscr{A}]$, there are functions $h$ and $k \in \mathscr{A}$ such that $Z(f) \cap Z(k)=\phi, \quad Z(g) \cap Z(h)=\phi$, and $Z(h) \cup Z(k)=X$. This yields $x \in Z(g) \subseteq X-Z(h) \subseteq X-Z(f) \subseteq A$. So $Z(k)$ is a neighborhood of $x$ and $Z(k) \subseteq A$.

3.12. Corollary. If $\mathscr{A}$ is a Wallman ring on $X$, then the weak topology generated by $\mathscr{A}$ is the given topology on $X$.

In the case of the Wallman ring $C^{*}(X)$ it is known that its Wallman compactification $\beta X$ is connected if and only if $C^{*}(X)$ cannot be expressed as the direct sum of nontrivial ideals. With slight modifications, this theorem can be generalized to arbitrary Wallman rings.

3.13. TheOREM. If $\mathscr{A}$ is a Wallman ring such that $\mathscr{A}=\mathscr{B} \oplus \mathscr{C}$ where $\mathscr{B}$ and $\mathscr{C}$ are proper ideals of $\mathscr{A}$ and if

$$
\begin{aligned}
& \mathscr{A}^{\prime}=\left\{\frac{f}{g}: f, g \in \mathscr{A}, \quad Z(g)=\phi\right\} \\
& \mathscr{B}^{\prime}=\left\{\frac{f}{g}: f \in \mathscr{B}, \quad g \in \mathscr{A}, \quad Z(g)=\phi\right\} \\
& \mathscr{C}^{\prime}=\left\{\frac{f}{g}: f \in \mathscr{C}, g \in \mathscr{A}, Z(g)=\phi\right\}
\end{aligned}
$$

then $\mathscr{B}^{\prime}$ and $\mathscr{C}^{\prime}$ are proper ideals of $\mathscr{A}$ and $\mathscr{A}^{\prime}=\mathscr{B}^{\prime} \oplus \mathscr{C}^{\prime}$.

3.14. Theorem. If $\mathscr{A}$ is a Wallman ring such that $\mathscr{A}=\mathscr{B} \oplus \mathscr{C}$ where $\mathscr{B}$ and $\mathscr{C}$ are proper ideals of $\mathscr{A}$, then $w(Z[\mathscr{A}]$ is disconnected.

Proof. Define $\mathscr{A}^{\prime}, \mathscr{B}^{\prime}$ and $\mathscr{C}^{\prime}$ as in the previous theorem. Then $1 \in \mathscr{A}^{\prime}$ implies there are function $f \in \mathscr{B}^{\prime}$ and $g \in \mathscr{C}^{\prime}$ such that $1=f+g$ 
and $f g=0$. Since $\mathscr{B}^{\prime}$ and $\mathscr{C}^{\prime}$ are proper ideals, they contain no functions whose zero sets are empty. In particular $Z(f) \neq \phi$ and $Z(g) \neq \phi$.

Now $Z(f) \cap Z(g)=Z\left(f^{2}+g^{2}\right)$

$$
\begin{aligned}
& =Z\left(f^{2}+2 f g+g^{2}\right) \\
& =Z(1) \\
& =\phi
\end{aligned}
$$

and $Z(f) \cup Z(g)=Z(f g)=X$.

Therefore $\mathrm{Cl}_{w\left(Z\left[\mathscr{A}^{\prime}\right]\right)} Z(f) \cap \mathrm{Cl}_{w\left(Z\left[\mathscr{A}^{\prime}\right]\right)} Z(g)=\phi$ and

$$
\mathrm{Cl}_{w\left(Z\left[\mathscr{A}^{\prime}\right]\right)} Z(f) \cup \mathrm{Cl}_{w\left(Z\left[\mathscr{A}^{\prime}\right]\right)} Z(g)=w\left(Z\left[\mathscr{A}^{\prime}\right]\right)
$$

where $\mathrm{Cl}_{w\left(Z\left[\mathscr{A}^{\prime}\right]\right)} Z(f)$ and $\mathrm{Cl}_{w\left(Z\left[\mathscr{A}^{\prime}\right]\right)} Z(g)$ are nonempty closed sets in $w\left(Z\left[\mathscr{A}^{\prime}\right]\right)$. This means $w\left(Z\left[\mathscr{A}^{\prime}\right]\right)$ is disconnected. Therefore $w(Z[\mathscr{A}])$ is disconnected, since it is homeomorphic to $W\left(Z\left[\mathscr{A}^{\prime}\right]\right)$.

Whether or not the converse of this theorem is valid is an open question. We do however have a partial converse.

3.15. Definition. If $\mathscr{A} \subseteq C(X)$, then $\mathscr{A}$ is sectionally replete if it satisfies the following condition:

$$
\text { If } \quad X=\bigcup_{i=1}^{n} Y_{i},
$$

where $Y_{1} \in Z[\mathscr{A}]$ for $i=1, \cdots, n$ and if there are functions $g_{i} \in \mathscr{A}$ such that $f\left|Y_{\imath}=g_{i}\right| Y_{\imath}$, for $i=1, \cdots, n$, then $f \in \mathscr{A}$.

3.16. THEOREM. If $\mathscr{A}$ is a sectionally replete Wallman ring and $w(Z[\mathscr{A}])$ is disconnected, then there are proper ideals $\mathscr{B}$ and $\mathscr{C}$ of $\mathscr{A}$ such that $\mathscr{A}=\mathscr{B} \oplus \mathscr{C}$.

Proof. Since $w(Z[\mathscr{A}])$ is disconnected, there exist nonempty, disjoint closed subsets $H$ and $L$ of $w(Z[\mathscr{A}])$ such that $H \cup L=$ $w(Z[\mathscr{A}])$. Therefore, there exist sets $B, C \in Z[\mathscr{A}]$ such that $H \subseteq$ $\mathrm{Cl}_{w(Z[\mathscr{A}])} B, L \subseteq \mathrm{Cl}_{w(Z[\mathscr{A}])} C$ and $B \cap C=\phi$ (Bentley [4]). Let $\mathscr{B}=\{f \in \mathscr{A}$ : $f=0$ on $B\}$ and $\mathscr{C}=\{f \in \mathscr{A}: f=0$ on $C\}$. $\mathscr{B}$ and $\mathscr{C}$ are ideals of $\mathscr{A}$ whose intersection is the zero ideal.

For each $f \in \mathscr{A}$ let

$$
f_{1}(x)=\left\{\begin{array}{c}
0 \quad \text { if } \quad x \in B \\
f(x) \quad \text { if } \quad x \in C
\end{array}\right.
$$

and

$$
f_{2}(x)=\left\{\begin{array}{c}
f(x) \text { if } \quad x \in B \\
0 \text { if } x \in C
\end{array}\right.
$$


Since $\mathscr{A}$ is sectionally replete $f_{1}$ and $f_{2} \in \mathscr{A} . \quad f=f_{1}+f_{2}, f_{1} \in \mathscr{B}$, and $f_{2} \in \mathscr{C}$. Therefore $\mathscr{A}=\mathscr{B} \oplus \mathscr{C}$.

The final topic in this section is the sublattice problem. The question of when a sublattice of $C(X)$ is a Wallman ring was answered by Biles [8]. Any sublattice of $C(X)$ whose zero sets form a base for the closed sets of $X$ is a Wallman ring. It is well-known that every closed subring of $C(X)$ is a closed sublattice of $C(X)$. So every uniformly closed Wallman ring is a sublattice of $C(X)$.

A question related to this, namely, "Given an arbitrary Wallman ring, is it possible to construct an equivalent Wallman ring which is a sublattice of $C(X)$ ?" does not appear to be easily answered.

Along this line we do have the following results.

3.17. THEOREM. Let $\mathscr{A}$ be a Wallman ring on $X$, let $\mathscr{B}$ be the ring generated by $\{|f|: f \in \mathscr{A}\}$. Then $\mathscr{B}$ is a Wallman ring on $X$ and $\mathscr{A} \cong \mathscr{B}$. $Z[\mathscr{B}]$.

Proof. $Z(|f| \pm|g|)=Z\left(f^{2} \pm g^{2}\right)$ and $Z(|f||g|)=Z(f g)$ so $Z[\mathscr{A}]=$

Clearly $\mathscr{B}$ is not necessarily a sublattice of $C(X)$. With further hypotheses we can get a little closer to a sublattice of $C(X)$.

3.18. Theorem. Let $\mathscr{A}$ be a Wallman ring over $X$, let $1 \in \mathscr{A}$, and let $Z(f \wedge 0) \in Z[\mathscr{A}]$ for each $f \in \mathscr{A}$. Then

$$
\mathscr{B}=\left\{\sum_{i=1}^{n} f_{i}\left|g_{i}\right|: \quad f_{i}, g_{i} \in \mathscr{A}, \quad n \in \mathcal{N}\right\}
$$

is a Wallman ring on $X, \mathscr{A} \cong \mathscr{B}$, and $\mathscr{B}$ contains the supremum and infimum of any two functions from $\mathscr{A}$.

Proof. (1) $Z[\mathscr{A}] \subseteq Z[\mathscr{B}]$ since $\mathscr{A} \subseteq \mathscr{B}$.

(2) $Z[\mathscr{B}] \subseteq Z[\mathscr{A}]$ since

$$
Z\left(\sum_{i=1}^{n} f_{i}\left|g_{i}\right|\right)=\cup\left\{P_{\alpha}: \alpha \subseteq\{1, \cdots, n\}\right\}
$$

where

$$
\begin{aligned}
S_{\alpha} & =\bigcap_{i \in \alpha} Z\left(g_{i} \wedge 0\right), \quad T_{\alpha}=\bigcap_{i \notin \alpha} Z\left(\left(-g_{i}\right) \wedge 0\right), \\
V_{\alpha} & =Z\left(\sum_{i \in \alpha} f_{i} g_{i}-\sum_{i \notin \alpha} f_{i} g_{i}\right)
\end{aligned}
$$


and

$$
P_{\alpha}=S_{\alpha} \cap T_{\alpha} \cap V_{\alpha}
$$

(3) $\mathscr{B}$ is a subring of $C(X)$.

Therefore $\mathscr{B}$ is a Wallman ring on $X$ and $\mathscr{B} \cong \mathscr{A}$.

(4) If $f, g \in \mathscr{A}$, then

$$
f \wedge g=g+[(f-g) \wedge 0]=\frac{f+g-|f-g|}{2}
$$

and

$$
f \vee g=g+[(f-g) \vee 0]=\frac{f+g+|f+g|}{2} .
$$

So $f \wedge g$ and $f \vee g \in \mathscr{B}$.

The following example illustrates that in trying to find a sublattice of $C(X)$ which is a Wallman ring equivalent to a given Wallman ring we cannot in general look at the sublattice generated by our given Wallman ring.

3.19. Example. Let $Y=[-1,1], X=Y-\{0\}, \mathcal{M}=\{f \in C(X)$ : for some compact set $H \subseteq X, f$ is constant on $X-H\}, j: X \rightarrow R$ be the inclusion map, $\mathscr{A}$ be the subring of $C(X)$ generated by $\mathcal{M} \cup\{j\}$, and $\mathscr{B}$ be the sublattice generated by $\mathscr{A}$. Then (1) $Z[\mathscr{A}]=Z[\mathcal{M}]$ so $\mathscr{A}$ is a Wallman ring and (2) $Z[\mathscr{B}] \not Z[\mathscr{A}]$.

Proof. (1) $\mathscr{A}=\left\{\sum_{n=0}^{m} g_{n} j^{n}: g_{n} \in \mathcal{M}, m \in \mathcal{N}\right\}, \quad$ and $\quad \mathcal{M} \subseteq \mathscr{A} \quad$ so $Z[\mathcal{M}] \subseteq Z[\mathscr{A}]$.

To show $Z[\mathscr{A}] \subseteq Z[\mathcal{M}]$ let $f \in \mathscr{A}, f=\sum_{n=0}^{m} g_{n} j^{n}$ where $g_{n} \in \mathcal{M}$. If $m=0$, then $f=g_{0} \in \mathcal{M}$ and we are through. Therefore suppose $m>0$.

For $n=0, \cdots, m$, there exist compact subsets $H_{n}$ of $X$ such that $g_{n}$ is constant on $X-H_{n}$.

Let $t>0$ such that $[-t, t] \subseteq Y-\cup_{n=0}^{m} H_{n}$. Let $a_{n}=g_{n}(t)$ for $n=0, \cdots, m$. Then $a_{n}=g_{n}(x)$ for $x \in[-t, t] \cap X, n=0, \cdots, m$. For $x \in[-t, t] \cap X, f(x)=\sum_{n=0}^{m} g_{n}(x) j^{n}(x)=\sum_{n=0}^{m} a_{n} x^{n}$ which is a polynomial over $[-t, t] \cap X$. So $f$ has finitely many zeros in $[-t, t] \cap X$. $Z(f)=[Z(f) \cap([-1,-t] \cup[t, 1]) \cup[Z(f) \cap[-t, t]]$ so $Z(f)$ is compact. Therefore $Z(f) \in Z[M]$.

(2) Suppose $Z[\mathscr{B}] \leqq Z[\mathscr{A}]=Z[\mathcal{M}]$. Let

$$
j^{+}=j \vee 0, \text { and } j^{-}=(-j)^{+} \text {. }
$$


Then $j^{+}$and $j^{-} \in \mathscr{B}$, and $Z\left(j^{+}\right) \cap Z\left(j^{-}\right)=\phi$. Now since $Z[\mathscr{B}] \leqq Z[\mathcal{M}]$ there exist functions $f, g \in M$ such that $Z\left(j^{+}\right) \subseteq Z(f), Z\left(j^{-}\right) \subseteq Z(g)$, and $Z(f) \cap Z(g)=\phi$. But $Z\left(j^{+}\right)=[-1,0) \cdot$ and $Z\left(j^{-}\right)=(0,1]$. So $\mathrm{Cl}_{Y}[-1,0) \cap \mathrm{Cl}_{Y}(0,1] \subseteq \mathrm{Cl}_{Y} Z(f) \cap \mathrm{CL}_{Y} Z(g)=\phi$ since $Y \cong w(Z[M])$. But $\mathrm{Cl}_{Y}[-1,0) \cap \mathrm{Cl}_{Y}(0,1]=\{0\}$ so we have a contradiction and it follows that $Z[\mathscr{B}] \not \equiv Z[\mathscr{A}]$.

Henriksen and Isbell [16] showed that $\mathscr{B}$ is a ring but we do not know if it is a Wallman ring.

This example shows us that there is a Wallman ring $\mathscr{A}$ such that if $\mathscr{C}$ is a Wallman ring on $X, \mathscr{C}$ is a sublattice of $C(X)$ and $\mathscr{A} \subseteq \mathscr{C}$ then $\mathscr{A}$ and $\mathscr{C}$ are not equivalent, since any such Wallman ring would contain $\mathscr{B}$.

Example 3.19 eliminates the obvious procedure for attacking another problem. Given an arbitrary Wallman ring we have shown there is an equivalent Wallman ring which contains all the rational constants. If we could state that the uniform closure of a Wallman ring is a Wallman ring equivalent to the original, we would have shown that every Wallman ring is equivalent to one which contains all the real constants. However, a uniformly closed subring of $C(X)$ is a sublattice of $C(X)$ and by example 3.19 there is a Wallman ring which is not contained in any equivalent Wallman ring which is a sublattice of $C(X)$. This means that in general the answer to getting the real constants in a Wallman ring does not lie in taking uniform closures. For now, we cannot answer the question, "For an arbitrary Wallman ring, is there an equivalent Wallman ring which contains all the real valued constant functions?"

\section{Examples of Wallman rings on locally compact} spaces. In this section, we present a method for constructing examples of Wallman Rings on locally compact spaces. These Wallman rings are determined by compactifications of the space which can be mapped by a certain kind of retract map onto the remainder. H. L. Bentley [6] has shown that these compactifications are Wallman; we show how they arise from Wallman rings.

Throughout this section, $X$ is assumed to be a locally compact space.

4.1. Definition. A closed subset $L$ of $X$ is called co-compact provided $\mathrm{Cl}_{Y}(X-L)$ is compact.

4.2. Definition. If $\mathscr{H}$ is a family of closed subsets of $X$, then the compact modification of $\mathscr{H}$ is the family

$C M(\mathscr{H})=\{(H \cap L) \cup B: H \in \mathscr{H}, L$ is co-compact, and $B$ is compact $\}$. 
4.3. Definition. (Borsuk [10].) A continuous map $f: Y \rightarrow K$ is called a retract map provided $K$ is a subspace of $Y$ and $f(x)=x$ for each $x \in K$.

4.4. Definition. (Bentley [6].) If $Y$ is a compactification of $X$, $K=Y-X$, and $f: Y \rightarrow K$ is a continuous map, then $f$ maps onto $K$ at $\infty$ provided $f[L]=K$ for each co-compact subset $L$ of $X$.

4.5. TheOREM. (Bentley [6].) Let $Y$ be a compactification of the locally compact space $X$, let $K=Y-X$, and let $f: Y \rightarrow K$ be a retract map which maps onto $K$ at $\infty$. Let $\mathscr{H}=\left\{X \cap f^{-1}(E): E\right.$ is a closed subset of $K\}$. Then $C M(\mathscr{H})$ is a normal base on $X$ and $w(C M(\mathscr{H})) \cong Y$.

Our objective is to exhibit a Wallman ring $\mathscr{A}$ on $X$ for which $w(Z[\mathscr{A}]) \cong Y$, with $Y$ as in the preceding theorem.

4.6. THEOREM. Let $Y$ be a compactification of the locally compact space $X$, let $K=Y-X$ and let $f: Y \rightarrow K$ be a retract map which maps onto $K$ at $\infty$. Let $\mathscr{B}$ be the set of all $h \in C(Y)$ for which there exists a co-compact set $L \subseteq X$ such that for all $z \in K, h\left[L \cap f^{-1}(\{z\})\right]=$ $\{h(z)\}$. Let $\mathscr{A}$ be the set of all restrictions $h \mid X$ with $h \in \mathscr{B}$. Then $\mathscr{A}$ is a Wallman ring on $X$ and $w(Z[\mathscr{A}]) \cong Y$.

The proof of this theorem will depend on the following lemmas. Let $Y, K, f, \mathscr{B}$ and $\mathscr{A}$ be as in Theorem 4.6 and let $\mathscr{H}$ be as in Theorem 4.5 .

\subsection{Lemma. $Z[\mathscr{A}] \leqq C M(\mathscr{H})$}

Proof. Let $h \in \mathscr{A}$, then $h$ has an extension $h^{\prime} \in C(Y)$ such that for some co-compact set $L$ in $X$

$$
h^{\prime}\left[L \cap f^{-1}(\{z\})\right]=h^{\prime}(z) \text { for all } z \in K .
$$

So $Z(h)=Z\left(h^{\prime}\right) \cap X$.

$$
\begin{aligned}
& =\left[Z\left(h^{\prime}\right) \cap \mathrm{Cl}_{X}(X-L)\right] \cup\left[Z\left(h^{\prime}\right) \cap L\right] . \\
& =\left[Z\left(h^{\prime}\right) \cap \mathrm{Cl}_{X}(X-L)\right] \cup\left[f^{-1}\left(Z\left(h^{\prime}\right) \cap K\right) \cap L\right] . \\
& =\left[Z\left(h^{\prime}\right) \cap \mathrm{Cl}_{X}(X-L)\right] \cup\left[\left(f^{-1}\left(Z\left(h^{\prime}\right) \cap K\right) \cap X\right) \cap L\right] .
\end{aligned}
$$

Now $Z\left(h^{\prime}\right) \cap \mathrm{Cl}_{X}(X-L)$ is compact, $L$ is co-compact, and $Z\left(h^{\prime}\right) \cap K$ is a closed subset of $K$, so $Z(h) \in C M(\mathscr{H})$ and $Z[\mathscr{A}] \leqq C M(\mathscr{H})$.

4.8. Lemma. $C M(\mathscr{H}) \leqq Z[\mathscr{A}]$. 
Proof. Let $F_{1}, F_{2} \in C M(\mathscr{H})$ such that $F_{1} \cap F_{2}=\phi$. There are sets $L_{1}$ and $L_{2}$ which are closed and co-compact in $X$, subsets $B_{1}$ and $B_{2}$ of $X$ which are compact, and subsets $E_{1}$ and $E_{2}$ of $K$ which are compact, such that $F_{i}=\left(L_{i} \cap f^{-1}\left(E_{i}\right)\right) \cup B_{i}$, for $i=1,2$. Also $F_{1} \cap F_{2}=\phi$ implies $\left[L_{1} \cap f^{-1}\left(E_{1}\right)\right] \cap\left[L_{2} \cap f^{-1}\left(E_{2}\right)\right]=\phi$. So have $L_{1} \cap L_{2} \cap f^{-1}\left(E_{1} \cap E_{2}\right)=\phi$. Since $f$ maps onto $K$ at $\infty$, and $L_{1} \cap L_{2}$ is co-compact, we conclude $E_{1} \cap E_{2}=\phi$.

$K$ is completely regular so there exists $u_{1}, u_{2} \in C^{*}(K)$, $u_{1}: K \rightarrow[0,1], u_{2}: K \rightarrow[0,1]$, such that $E_{1} \subseteq Z\left(u_{1}\right), E_{2} \subseteq Z\left(u_{2}\right)$, and $Z\left(u_{1}\right) \cap Z\left(u_{2}\right)=\phi$. Let $f_{1}^{\prime}=u_{1} \circ f$, and $f_{2}^{\prime}=u_{2} \circ f$. Then $f_{1}^{\prime}, f_{2}^{\prime} \in C(Y)$, $f_{1}^{\prime}: Y \rightarrow[0,1], f_{2}^{\prime}: Y \rightarrow[0,1]$.

If $x \in L_{i} \cap f^{-1}\left(E_{i}\right)$, then $f(x) \in E_{i}$; and since $u_{i}=0$ on $E_{i}$, it follows that $x \in Z\left(f_{i}^{\prime}\right.$ for $i=1,2$. Also $Z\left(f_{i}^{\prime}\right) \cap Z\left(f_{2}^{\prime}\right)=\phi$.

Now $\left\{\left[L_{1} \cap f^{-1}\left(E_{1}\right)\right] \cup B_{1}\right\} \cap\left\{\left[L_{2} \cap f^{-1}\left(E_{2}\right)\right] \cup B_{2}\right\}=\phi$ and these two sets are elements of a normal base for $Y$ so

$$
\mathrm{Cl}_{Y}\left\{\left[L_{1} \cap f^{-1}\left(E_{1}\right)\right] \cup B_{1}\right\} \cap \mathrm{Cl}_{Y}\left\{\left[L_{2} \cap f^{-1}\left(E_{2}\right)\right] \cup B_{2}\right\}=\phi .
$$

Therefore there exist closed sets $G_{1}$ and $G_{2}$ in $Y$ such that $\mathrm{Cl}_{Y}\left\{\left[L_{i} \cap\right.\right.$ $\left.\left.f^{-1}\left(E_{i}\right)\right] \cup B_{i}\right\} \subseteq \operatorname{Int}_{Y} G_{i}$, for $i=1,2 . B_{1}$ and $B_{2}$ are disjoint compact sets in $X$, so there are disjoint compact sets $C_{1}$ and $C_{2}$ in $X$ such that $B_{i} \subseteq$ Int $_{X} C_{i}, i=1,2$. Then $C_{i} \cap G_{i}$ is a compact set in $X$ which contains $B_{i}$ in its interior and is disjoint from $\left[C_{j} \cap G_{j}\right] \cup\left[L_{j} \cap f^{-1}\left(E_{j}\right)\right]$ for $i \neq j$; $i=1,2 ; j=1,2$.

Now define $h_{1}=0$ on $B_{1}, h_{1}=1$ on $\mathrm{Cl}_{Y}\left(Y-\left(C_{1} \cap G_{1}\right)\right)$. These are disjoint closed sets in $Y$ so we can take $h_{1}$ to be a continuous function on $Y, h_{1}: Y \rightarrow[0,1]$. Similarly define $h_{2}: Y \rightarrow[0,1], h_{2}=0$ on $B_{2}, h_{2}=1$ on $\mathrm{Cl}_{Y}\left(Y-\left(C_{2} \cap G_{2}\right)\right)$. So $Z\left(h_{1}\right) \cap Z\left(h_{2}\right)=\phi$.

Now we have no assurance that $Z\left(f_{2}^{\prime}\right) \cap Z\left(h_{1}\right)=\phi$ or that $Z\left(f_{1}^{\prime}\right) \cap Z\left(h_{2}\right)=\phi$ so we modify $f_{1}^{\prime}$ and $f_{2}^{\prime}$.

$f^{-1}\left(Z\left(u_{1}\right)\right) \cap\left(C_{2} \cap G_{2}\right)$ is a compact set in $X$ disjoint from $f^{-1}\left(E_{1}\right) \cap$ $L_{1}$, a closed set in $X$. Therefore since $X$ is locally compact there exists a compact subset $D_{1}$ of $X$ such that

$$
f^{-1}\left(Z\left(u_{1}\right)\right) \cap C_{2} \cap G_{2} \subseteq \operatorname{Int}_{X} D_{1} \subseteq D_{1} \subseteq X-\left(f^{-1}\left(E_{1}\right) \cap L_{1}\right) .
$$

Similarly there exists a compact subset $D_{2}$ of $X$ such that

$$
f^{-1}\left(Z\left(u_{2}\right)\right) \cap C_{1} \cap G_{1} \subseteq \operatorname{Int}_{X} D_{2} \subseteq D_{2} \subseteq X-\left(f^{-1}\left(E_{2}\right) \cap L_{2}\right) .
$$

Since $f^{-1}\left[Z\left(u_{1}\right)\right] \cap C_{2} \cap G_{2}$ and $\mathrm{Cl}_{Y}\left(Y-D_{1}\right)$ are disjoint closed subsets of $Y$ and since $Y$ is compact, hence normal, there exists a 
continuous function $g_{1}: Y \rightarrow[0,1]$ such that $g_{1}$ is 1 on $f^{-1}\left[Z\left(u_{1}\right) \cap C_{2} \cap\right.$ $G_{2}$ ] and $g_{1}$ is 0 on $\mathrm{Cl}_{Y}\left(Y-D_{1}\right)$. Similarly define $g_{2}$.

Now let $f_{1}=f_{1}^{\prime} \vee g_{1}$ and $f_{2}=f_{2}^{\prime} \vee g_{2}$. Then $f_{1}, f_{2} \in C(Y)$, $f_{1}: Y \rightarrow[0,1], f_{2}: Y \rightarrow[0,1], Z\left(f_{1}\right) \cap Z\left(f_{2}\right)=\phi, Z\left(f_{1}\right) \cap Z\left(h_{2}\right)=\phi$ and $Z\left(f_{2}\right) \cap Z\left(h_{1}\right)=\phi$.

Finally let $\alpha_{1}=f_{1} \wedge h_{1}$ and $\alpha_{2}=f_{2} \wedge h_{2} . \quad F_{1} \subseteq Z\left(\alpha_{1}\right)$ since

$$
f^{-1}\left(E_{1}\right) \cap L \subseteq Z\left(f_{1}^{\prime}\right) \cap Z\left(g_{1}\right)=Z\left(f_{1}\right) \subseteq Z\left(\alpha_{1}\right)
$$

and $B_{1} \subseteq Z\left(h_{1}\right) \subseteq Z\left(\alpha_{1}\right)$. Similarly $F_{2} \subseteq Z\left(\alpha_{2}\right) . \quad Z\left(\alpha_{1}\right)$ and $Z\left(\alpha_{2}\right)$ are disjoint since:

$$
\begin{aligned}
Z\left(\alpha_{1}\right) \cap Z\left(\alpha_{2}\right)= & \left(Z\left(f_{1}\right) \cup Z\left(h_{1}\right)\right) \cap\left(Z\left(f_{2}\right) \cup Z\left(h_{2}\right)\right) \\
= & \left(Z\left(f_{1}\right) \cap Z\left(f_{2}\right)\right) \cup\left(Z\left(f_{1}\right) \cap Z\left(h_{2}\right)\right) \cup\left(Z\left(h_{1}\right) \cap Z\left(f_{2}\right)\right) \\
& \times \cup\left(Z\left(h_{1}\right) \cap Z\left(h_{2}\right)\right) \\
= & \phi
\end{aligned}
$$

$\alpha_{1} \in \mathscr{B}$ since $\alpha_{1}=f_{1}^{\prime}$ on $\mathrm{Cl}_{Y} L_{1} \cap \mathrm{Cl}_{Y}\left(Y-D_{1}\right) \cap \mathrm{Cl}_{Y}\left(Y-\left(C_{1} \cap G_{1}\right)\right)$, a set whose intersection with $X$ is co-compact. Similarly $\alpha_{2} \in \mathscr{B}$.

Therefore we have functions $\alpha_{1} \mid X$ and $\alpha_{2} \mid X$ whose zero sets separate $F_{1}$ and $F_{2}$, consequently $C M(\mathscr{H}) \leqq Z[\mathscr{A}]$.

\subsection{Lemma. $Z[\mathscr{A}]$ is a lattice of closed subsets of $X$.}

Proof. If $f, g \in \mathscr{B}$, and $L_{f}$ and $L_{g}$ are the co-compact sets associated with $f$ and $g$ in the definition of $\mathscr{B}$ then $f-g$ and $f g$ satisfy the condition for being elements of $\mathscr{B}$ on the co-compact set $L_{f} \cap L_{g}$. Consequently $\mathscr{B}$ is a subring of $C(Y)$ so $Z(\mathscr{B})$ is a lattice of closed subsets of $Y$ and hence $Z(\mathscr{A})$ is a lattice of closed subsets of $X$.

We are now in a position to prove Theorem 4.6.

Proof. By Lemmas 4.7 and $4.8 Z[\mathscr{A}] \cong C M(\mathscr{H})$ and by Lemma $4.9, Z[\mathscr{A}]$ is a lattice of closed subsets of $X$. Therefore by Theorem 2.12 $Z[\mathscr{A}]$ is a normal base on $X$ and $w(Z[\mathscr{A}]) \cong w(C M(\mathscr{H}))$. But $w(C M(\mathscr{H})) \cong Y$, so we have $w(Z[\mathscr{A}]) \cong Y$.

We note that the theorem is also valid when $f$ is defined just on the closure in $Y$ of some co-compact subset of $X$.

The following corollary gives a Wallman ring which generates the one point compactification of a locally compact space. This result was earlier observed by Brooks [11]. 
4.10. Corollary. If $X$ is a locally compact space, then $\mathscr{A}=$ $\{f \in C(X)$ : there is a co-compact subset of $X$ on which $f$ is constant $\}$ is a Wallman ring on $X$ and $w(Z[\mathscr{A}])$ is the Alexandroff compactification of $X$.

Proof. Let $Y$ be the Alexandroff compactification of $X$. There can be only one function mapping $Y$ onto $(Y-X)$ since there is just one point in $(Y-X)$. Clearly this function maps onto $(Y-X)$ at $\infty$. Therefore the hypotheses of Theorem 4.6 are satisfied. By examining the set $\mathscr{B}$ as defined in the theorem, we see $\mathscr{B}=\{h \in C(Y): h(L)$ is constant for some co-compact subset $L$ of $X\}$. Consequently $\mathscr{A}$ is a Wallman ring which generates the Alexandroff compactification of $X$.

The zero sets of this Wallman ring are precisely those zero sets of $X$ which are either compact or co-compact.

4.11. Theorem. If $X$ is a locally compact space and $\mathscr{A}=$ $\{f \in C(X)$ : there is a co-compact subset of $X$ on which $f$ is constant $\}$, then $Z[\mathscr{A}]=\{F: F \in Z(X)$ and $F$ is either compact or co-compact $\}$.

Proof. Let $f \in \mathscr{A}$, then $Z(f) \in Z(X)$. Let $F$ be the co-compact set on which $f$ is constant. Then $Z(f) \cap F=\phi$ or $F \subseteq Z(f)$. If $Z(f) \cap F=$ $\phi$, then $Z(f) \subseteq \mathrm{Cl}_{X}(X-F)$ which implies $Z(f)$ is compact. If $F \subseteq Z(f)$, then $\mathrm{Cl}_{X}(X-Z(f)) \subseteq \mathrm{Cl}_{X}(X-F)$ which implies that $\mathrm{Cl}_{X}(X-Z(f))$ is compact and $Z(f)$ is co-compact.

Now let $Z(f)$ be a zero set of $X$ which is either compact or co-compact, $f \in C(X)$. If $Z(f)$ is compact, then let $f^{\prime}=1 \wedge|f|$ Then $Z(f)=Z\left(f^{\prime}\right)$. Since $Z(f)$ is compact and $X$ is locally compact there is a compact subset $W$ of $X$ such that $Z(f) \subseteq$ Int $W \subseteq W \subseteq X$. Also there is a function $g \in C(X)$ such that $g[Z(f)]=\{0\}, \quad g[X-$ Int $W]=\{1\}$, $g: X \rightarrow[0,1]$. Define $h=f^{\prime} \vee g$. Then $Z(h)=Z(f)$ and $h$ is constant on $(X-$ Int $W)$ which is a co-compact subset of $X$. Therefore $Z(f) \in$ $Z[\mathscr{A}]$.

If $Z(f)$ is co-compact, then $f$ is constant on the co-compact set $Z(f)$, so $f \in \mathscr{A}$, and $Z(f) \in Z[\mathscr{A}]$.

We will now define a Wallman ring $\mathscr{A}$, on the open unit disc such that $w(Z[\mathscr{A}])$ is the closed unit disc.

4.12. Example. Let $X$ be the open unit disc in Euclidean 2-space and let $Y$ be the closed unit disc.

We will consider the elements of $Y$ to be complex numbers. Let $K=\{z \in Y:|z|=1\}$, let $J=\left\{z \in Y:|z| \geqq \frac{1}{2}\right\}$ and define a function $f: J \rightarrow K$ by

$$
f(z)=\frac{z}{|z|} \text { for eaach } z \in J
$$


Let $\mathscr{B}$ be the set of all functions $h \in C(Y)$ for which there exists a co-compact subset $L$ of $X$ such that for all $z \in L \cap J, h(z)=h(z \| z \mid)$. Note that $\mathscr{B}$ can equivalently be described as the set of all functions $h \in C(Y)$ for which there exists $t \in\left[\frac{1}{2}, 1\right]$ such that for all $z$ with $t \leqq|z| \leqq 1, h(z)=h(z /|z|)$.

As was noted after the proof of Theorem 4.6, that theorem is valid if the retract map is defined only on the closure in $Y$ of some co-compact subset of $X$. In the present situation, $J$ is such a closure. Clearly, $f$ maps $J \cap X$ onto $K$ at $\infty$. Therefore if we let $\mathscr{A}=$ $\{h \mid X: h \in \mathscr{B}\}$, then $\mathscr{A}$ is a Wallman ring on $X$ which induces a compactification equivalent to the closed disc.

Acknowledgements. The authors would like to thank Professor Stuart A. Steinberg for some illuminating discussions and for suggestions for improvements in the manuscript. Thanks are also due to Professors Anne K. Steiner and Budmon Davis who suggested several improvements.

\section{REFERENCES}

1. J. M. Aarts, Every Metric Compactification is a Wallman Type Compactification, Proceedings of the International Symposium on Topology and its Applications, (1968), 29-34.

2. R. A. Alo, and H. L. Shapiro, Normal bases and compactifications, Math. Annalen, 175 (1968), 337-340.

3. B. Banaschewski, On Wallman's method of compactification, Math. Nachr., 27 (1963), 105-114.

4. H. L. Bentley, Normal Bases and Compactifications, Proceedings of the Topology Conference, Oklahoma (1972).

5. - Some Wallman compactifications determined by retracts, Trans. Amer. Math. Soc., 33 (1972), 587-593.

6. - Some Wallman compactifications of locally compact spaces, Fund. Math., 75 (1972), $13-24$.

7. E. S. Berney, On Wallman compactifications, Notices Amer. Math. Soc., 17 (1970), 215 A-672-462.

8. C. M. Biles, Gelfand and Wallman-Type compactifications, Pacific J. Math., 35 (1970), 267-278.

9. Wallman-type compactifications, Proc. Amer. Math. Soc., 25 (1970), 363-368.

10. K. Borsuk, Theory of Retracts, Polish Scientific Publishers, Warsaw, 1967.

11. R. M. Brooks, On Wallman compactifications, Fund. Math., 60 (1967), 157-173.

12. Orrin Frink, Compactifications and semi-normal spaces, Amer. J. Math., 86 (1964), 602-607.

13. L. Gillman, and M. Jerison, Rings of Continuous Functions, Van Nostrand, 1960.

14. A. W. Hager, On inverse-closed subalgebras of $C(X)$, Proc. London Math. Soc., (3) 19 (1969), 233-257.

15. A. W. Hager, and D. G. Johnson, A note on certain subalgebras of $C(X)$, Canadian J. Math., 20 (1968), 389-393.

16. N. Henrickesen, and J. Isbell, Lattice ordered rings and function rings, Pacific J. Math., 12 (1962), 533-565.

17. Olav Njastad, On Wallman-type compactifications, Math. Zeitzchr., 91 (1966), 267-276.

18. N. A. Sanin, On special extensions of topological spaces, Dokl, Akad. Nauk S.S.S.R., 38 (1943), 6-9.

19. E. F. Steiner, Wallman spaces and compactifications, Fund. Math., 61 (1968), 295-304. 
20. A. K. Steiner, and E. F. Steiner, Products of compact metric spaces are regular Wallman, Indag. Math., 30 (1968), 428-430.

21. W Wallman and Z-compactifications, Duke Math. J., 35 (1968), 269-276.

22. B. J. Taylor, and H. L. Bentley, The Stone-Weierstrass Theorem for Wallman Rings, preprint.

23. H. Wallman, Lattices and topological spaces, Ann. Math., 39 (1938), 112-126.

Received February 28, 1974.

iBM Corporation, Toledo, Ohio

AND

THE UNIVERSITY OF TOLEDO 



\section{CONTENTS}

Zvi Artstein and John A. Burns, Integration of compact set-valued functions

J. A. Beachy and W. D. Blair, Rings whose faithful left ideals are cofaithful

Mark Benard, Characters and Schur indices of the unitary reflection group $[321]^{3}$

H. L. Bentley and B. J. Taylor, Wallman rings ............................. 15

E. Berman, Matrix rings over polynomial identity rings II ............... 37

Simeon M. Berman, A new characterization of characteristic functions of absolutely continuous distributions ........................ 323

Monte B. Boisen, Jr. and Philip B. Sheldon, Pre-Prüfer rings ............ 331

A. K. Boyle and K. R. Goodearl, Rings over which certain modules are injective

J. L. Brenner, R. M. Crabwell and J. Riddell, Covering theorems for finite nonabelian simple groups. $V$

H. H. Brungs, Three questions on duo rings .................................... 345

Iracema M. Bund, Birnbaum-Orlicz spaces of functions on groups ....351

John D. Elwin and Donald R. Short, Branched immersions between 2-manifolds of higher topological type

J. K. Finch, The single valued extension property on a Banach space ................................................................................. 61

J. R. Fisher, A Goldie theorem for differentiably prime rings ............ 71

Eric M. Friedlander, Extension functions for rank 2, torsion free abelian groups ...................................................................... 371

J. Froemke and R. Quackenbusch, The spectrum of an equational class of groupoids

B. J. Gardner, Radicals of supplementary semilattice sums of

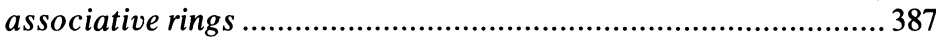

Shmuel Glasner, Relatively invariant measures ...............................393

G. R. Gordh, Jr. and Sibe Mardešić, Characterizing local connectedness in inverse limits...

S. Graf, On the existence of strong liftings in second countable topological spaces

S. Gudder and D. Strawther, Orthogonally additive and orthogonally increasing functions on vector spaces ........................................427

F. Hansen, On one-sided prime ideals .......................................... 79

D. J. Hartfiel and C. J. Maxson, A characterization of the maximal monoids and maximal groups in $\beta x$.

Robert E. Hartwig and S. Brent Morris, The universal flip matrix and the generalized faro-shuffle 


\section{Pacific Journal of Mathematics}

Vol. 58, No. 1

March, 1975

John Allen Beachy and William David Blair, Rings whose faithful left ideals are cofaithful .................................... 1

Herschel Lamar Bentley and Barbara June Taylor, Wallman rings ........ 15

Elizabeth Berman, Matrix rings over polynomial identity rings. II ...... 37

Ann K. Boyle and Kenneth R. Goodearl, Rings over which certain modules are injective ................................. 43

J. L. Brenner, Robert Myrl Cranwell and James Riddell, Covering theorems for finite nonabelian simple groups. $V \ldots \ldots \ldots \ldots \ldots \ldots \ldots \ldots \ldots$

James Kenneth Finch, The single valued extension property on a Banach

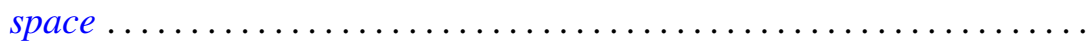

John Robert Fisher, A Goldie theorem for differentiably prime rings........ Friedhelm Hansen, On one-sided prime ideals .................... Jon Craig Helton, Product integrals and the solution of integral equations..........................................

Barry E. Johnson and James Patrick Williams, The range of a normal

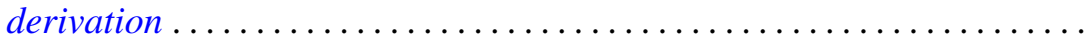

Kurt Kreith, A dynamical criterion for conjugate points ............ 123

Robert Allen McCoy, Baire spaces and hyperspaces .............. 133

John McDonald, Isometries of the disk algebra ................ 143

H. Minc, Doubly stochastic matrices with minimal permanents ......... 155

Shahbaz Noorvash, Covering the vertices of a graph by vertex-disjoint paths. ...

Theodore Windle Palmer, Jordan *-homomorphisms between reduced Banach*-algebras

Donald Steven Passman, On the semisimplicity of group rings of some

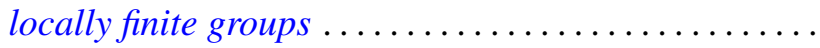

Mario Petrich, Varieties of orthodox bands of groups .

Robert Horace Redfield, The generalized interval topology on distributive

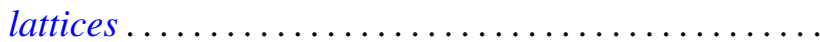

James Wilson Stepp, Algebraic maximal semilattices .... . .

Patrick Noble Stewart, A sheaf theoretic representation of rings with Boolean orthogonalities ........................

Ting-On To and Kai Wing Yip, A generalized Jensen's inequality......... 255

Arnold Lewis Villone, Second order differential operators with self-adjoint

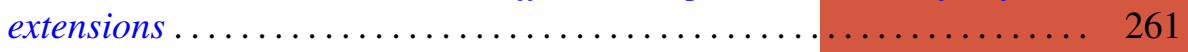

Martin E. Walter, On the structure of the Fourier-Stieltjes algebra ....... 267

John Wermer, Subharmonicity and hulls .................... 283

Edythe Parker Woodruff, A map of $E^{3}$ onto $E^{3}$ taking no disk onto a

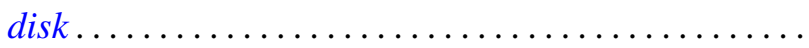

